\title{
Characterization of the Duffy-Binding-Like Domain of Plasmodium falciparum Blood-Stage Antigen 332
}

\author{
Sandra Nilsson, ${ }^{1}$ Kirsten Moll, ${ }^{1}$ Davide Angeletti, ${ }^{1}$ Letusa Albrecht, ${ }^{1}$ Inari Kursula, ${ }^{2,3}$ \\ Ning Jiang, ${ }^{4}$ Xiaodong Sun, ${ }^{5}$ Klavs Berzins, ${ }^{6}$ Mats Wahlgren, ${ }^{1}$ and Qijun Chen ${ }^{1,7}$ \\ ${ }^{1}$ Department of Microbiology, Tumor, and Cell Biology, Karolinska Institutet, 17177 Stockholm, Sweden \\ ${ }^{2}$ Department of Medical Biochemistry and Biophysics, Karolinska Institutet, 17177 Stockholm, Sweden \\ ${ }^{3}$ Centre for Structural Systems Biology, Helmholtz Centre for Infection Research and Deutsches Elektronen-Synchrotron, \\ University of Hamburg, 22607 Hamburg, Germany \\ ${ }^{4}$ Key Laboratory of Zoonosis, Ministry of Education, Jilin University, Changchun 130062, China \\ ${ }^{5}$ Institute of Parasitic Disease Control, Puer City, Yunnan Province, China \\ ${ }^{6}$ Department of Immunology, Wenner-Gren Institute, Stockholm University, 10691 Stockholm, Sweden \\ ${ }^{7}$ Laboratory of Parasitology, Institute of Pathogen Biology, Chinese Academy of Medical Sciences, Beijing 100730, China
}

Correspondence should be addressed to Sandra Nilsson, sandra.nilsson@ki.se and Mats Wahlgren, mats.wahlgren@ki.se

Received 25 March 2011; Accepted 8 June 2011

Academic Editor: Neena Valecha

Copyright (๑) 2011 Sandra Nilsson et al. This is an open access article distributed under the Creative Commons Attribution License, which permits unrestricted use, distribution, and reproduction in any medium, provided the original work is properly cited.

Studies on Pf332, a major Plasmodium falciparum blood-stage antigen, have largely been hampered by the cross-reactive nature of antibodies generated against the molecule due to its high content of repeats, which are present in other malaria antigens. We previously reported the identification of a conserved domain in Pf332 with a high degree of similarity to the Duffy-bindinglike (DBL) domains of the erythrocyte-binding-like (EBL) family. We here describe that antibodies towards Pf332-DBL are induced after repeated exposure to $P$. falciparum and that they are acquired early in life in areas of intense malaria transmission. Furthermore, a homology model of Pf332-DBL was found to be similar to the structure of the EBL-DBLs. Despite their similarities, antibodies towards Pf332-DBL did not display any cross-reactivity with EBL-proteins as demonstrated by immunofluorescence microscopy, Western blotting, and peptide microarray. Thus the DBL domain is an attractive region to use in further studies on the giant Pf332 molecule.

\section{Introduction}

Plasmodium falciparum malaria is a major human disease that accounts for 1-2 million deaths annually [1]. The disease affects predominantly children under the age of five, as older children and adults living in malaria endemic areas become immune against severe forms of the disease after being repeatedly exposed to the parasite.

During blood-stage development, $P$. falciparum parasites invade red blood cells (RBC) and cause them to sequester from the blood circulation by adhering to host endothelial cells. Both invasion and sequestration are central to the pathogenesis of malaria, and they require an adhesive cysteine-rich domain referred to as the Duffy-binding-like (DBL) domain. The DBL domains are present in two different protein families; the erythrocyte-binding-like (EBL) family of invasion proteins and the large and diverse P. falciparum erythrocyte membrane protein 1 (PfEMP1) family of cytoadherence proteins $[2,3]$. We recently reported the identification of a previously unknown domain in the $P$. falciparum antigen 332 with a high degree of similarity to the DBL domains of the EBL family [4]. Most EBL members are transmembrane proteins, which are expressed in schizonts and localize to merozoite micronemes from where they are released prior to or during host cell invasion [5]. In contrast, Pf332 is expressed in trophozoites and is later on cotransported together with the surface-destined PfEMP1 in parasite-induced membrane structures, referred to as Maurer's clefts $[6,7]$. In schizonts, Pf332 can be found in association with the RBC plasma membrane, although it 
is not clear whether the antigen becomes surface exposed or not $[4,7]$. Pf332 is a large protein of approximately $700 \mathrm{kDa}$ consisting predominantly of degenerate repeats rich in glutamic acid (Glu) [8-10]. Pf332 further lacks the typical EBL gene structure with the tandem DBL domains and the cysteine-rich region adjacent to the transmembrane domain [3]. In Pf332, the single DBL domain is located in the $\mathrm{N}$-terminus of the protein and is followed by a putative transmembrane region and a large number of negatively charged Glu-repeats, which make up a major part of the molecule (Figure 1(a)) [4]. In addition, Pf332 has a Cterminal tryptophan-rich region (WRD) with similarities to WRDs found in SURFINS, PkSICAvar, and PfEMP1 [11]. Although the function of Pf332 is not clear, the antigen is present in all isolates investigated $[4,10]$, indicating that it is of importance for the parasite.

Previous studies on Pf332 have primarily focused on EB200, a 157 amino-acid-long Glu-rich fragment, located in the central repeat region of the protein (Figure 1(a)) [9]. Individuals living in malaria endemic areas have been shown to have antibodies recognizing this region $[12,13]$ and these antibodies were associated with fewer clinical malaria attacks [14]. However, antibodies recognizing the repeat regions in Pf332 are possibly cross-reactive with other Glu-rich antigens in P. falciparum such as Pf155/RESA, making it difficult to ascertain the antibody specificity. Therefore, there is a need for a more specific Pf332 marker. The DBL domain of Pf332 is conserved between different parasite strains but with a distinct sequence, as compared to the DBL domains of the EBL family [4]. The conservation and distinct sequence of the DBL region makes it an attractive target for more detailed and specific studies on the molecule Pf332.

In the present study, we have expressed soluble Pf332DBL recombinant protein in Escherichia coli and analyzed its folding and secondary structure content by circular dichroism (CD) spectroscopy. We have further created a homology model of Pf332-DBL in order to assess structural similarities to the DBL domains of the EBL family. The predicted three-dimensional structure was found to have a fold similar to the DBL domains of $P$. falciparum EBA-175 and $P$. knowlesi Duffy-binding protein, which was in good agreement with the measured $\mathrm{CD}$ data. We have further examined naturally acquired antibodies towards the DBL domain of Pf332 and a DBL1 $\alpha$-domain from the variant surface antigen PfEMP1 [15], in order to get a better insight into the immunogenicity of the DBL domain of Pf332. We here show that Pf332 is widely recognized in distinct malaria endemic regions and that antibodies towards Pf332-DBL do not display any cross-reactivity with DBL-containing EBL proteins. This makes the DBL domain an attractive region to use in further studies on the Pf332 molecule.

\section{Methods}

2.1. Cloning, Expression, and Purification of Pf332-DBL and PfEMP1-DBL1 $\alpha$. The DBL1 $\alpha$-domain of var1FCR3S1.2 and the DBL domain of Pf332 were amplified with oligonucleotide primers (F-DBL FCR351.2 CCATGG CTA CTT CCG
GAG GA, R-DBL CTG CTT ATC; F-DBL 3 D7PF332 GCATGC GAA GCA ACA TCA ACA ACA AGG, R-DBL 3 D7Pf332 GGATCC GTA CTT CTT CTC GAA CAC C) from genomic DNA and cloned into the NcoI and BglII or SphI and BamHI restriction site of the pQE60 or pQE70 vector (Qiagen, Düsseldorf, Germany), respectively. His-tagged recombinant Pf332-DBL and var1FCR3S1.2-DBL1 $\alpha$ proteins were expressed according to standard procedures with modifications. Briefly, SG13009 E. coli (Qiagen) were grown in LB medium until $\mathrm{OD}_{600}$ reached $0.8-1.0$, and the culture was subsequently induced with $100 \mu \mathrm{M}$ IPTG and grown for an additional $3 \mathrm{~h}$ at room temperature. The bacteria were harvested by centrifugation at $990 \mathrm{~g}$ for $20 \mathrm{~min}$, after which the pellet was resuspended in washing buffer (20 mM HEPES (pH 7.4), $30 \mathrm{mM}$ imidazole, and $500 \mathrm{mM} \mathrm{NaCl}$ ). $1 \mathrm{mg} / \mathrm{mL}$ lysozyme (BioSite, San Diego, CA, USA) was added, to the lysate, which was incubated for 30 min on ice. Complete Cocktail protease inhibitors (Roche, Basel, Switzerland) were added and the cells were broken by sonication on ice. The lysate was clarified by centrifugation at $13250 \mathrm{~g}$ for $20 \mathrm{~min}$, after which the supernatant was collected and filtered through a $0.45 \mu \mathrm{m}$ filter.

For protein purification, a His GraviTrap column (GE Healthcare, Uppsala, Sweden) was used, according to the manufacturer's instructions. The cleared lysate was loaded onto the column and allowed to pass through twice. The column was washed extensively with washing buffer and the bound protein eluted with elution buffer containing $20 \mathrm{mM}$ HEPES (pH 7.4), $500 \mathrm{mM}$ imidazole, and $500 \mathrm{mM} \mathrm{NaCl}$. All eluted fractions of Pf332-DBL were analyzed by SDSPAGE using a $12 \%$ separation gel, and fractions containing the highest concentration of pure protein were pooled, dialyzed overnight against phosphate buffered saline (PBS), and finally concentrated using Centriprep centrifugal filter units (Millipore, Billerica, MA, USA). Possible aggregates of Pf332-DBL were thereafter spun down (5 min at $16060 \mathrm{~g})$ and the supernatant was applied to a $16 / 60$ Superdex 200 size exclusion chromatography column (GE Healthcare), equilibrated with $20 \mathrm{mM}$ HEPES ( $\mathrm{pH} 7.5$ ), $300 \mathrm{mM} \mathrm{NaCl}$, and $10 \%$ glycerol at a flow rate of $0.5 \mathrm{~mL} / \mathrm{min}$. The elution was followed by UV absorbance at $280 \mathrm{~nm}$, and the peak fractions were collected and analyzed by SDS-PAGE, as above. Fractions containing the highest concentration of pure protein were pooled and concentrated. The identity of the purified protein was confirmed by mass spectrometry using the Royal Institute of Technology (KTH, Stockholm, Sweden) mass spectrometry core facility.

2.2. Circular Dichroism Spectroscopy. Circular dichroism (CD) spectroscopy measurements of Pf332-DBL in PBS (pH 7.4) were conducted on a JASCO J-810 spectropolarimeter, using a cuvette with a $0.1 \mathrm{~cm}$ path length. The spectra were accumulated from eight scans at $20^{\circ} \mathrm{C}$, using a $0.1 \mathrm{~nm}$ data pitch, $1 \mathrm{~s}$ signal response time, and a $1 \mathrm{~nm}$ bandwidth. Data were recorded in the far UV region from 195 to $260 \mathrm{~nm}$. The scanning speed was $20 \mathrm{~nm} / \mathrm{min}$ and sensitivity $100 \mathrm{mdeg}$. The average spectra were corrected by subtracting the background spectrum measured for the buffer. 


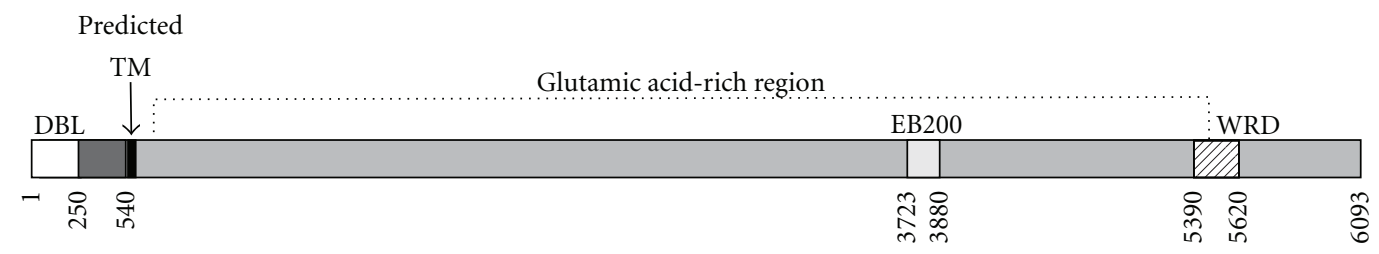

(a)

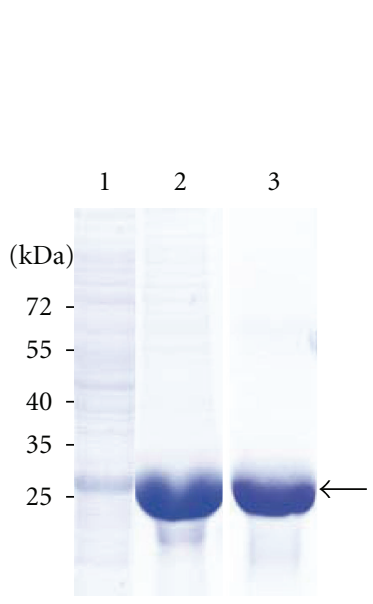

(b)

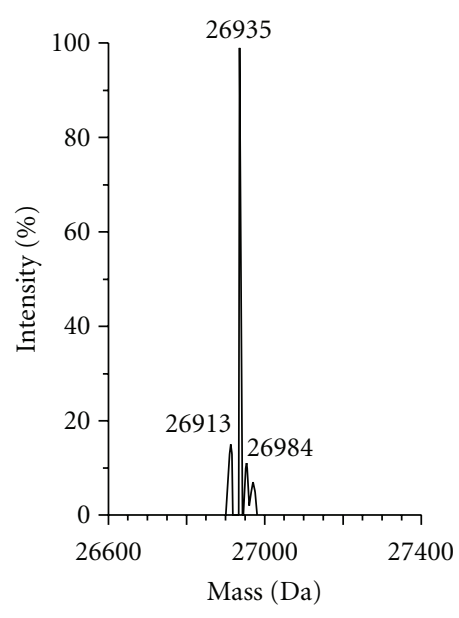

(c)

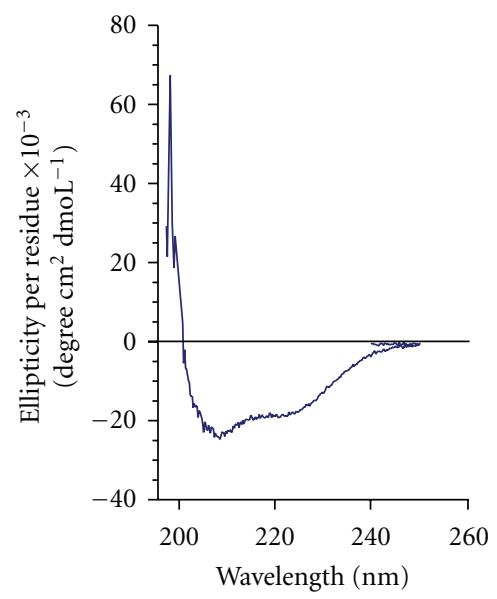

(d)

Figure 1: Purification and characterization of recombinant Pf332-DBL. (a) Schematic representation of the full-length Pf332 protein. Residues 1-570 are encoded by the first exon, which contains the Duffy-binding-like (DBL) domain (white) and the predicted transmembrane domain (black). Exon 2 encodes the extensive glutamic acid-rich repeat region (grey), which contains the repetitive EB200 region (light gray) and the tryptophan-rich WRD region (striped). (b) Coomassie-stained SDS polyacrylamide gel after each purification step of Pf332-DBL. Lane 1: E. coli soluble lysate, lane 2: after affinity purification on a Ni-column, and lane 3: after size exclusion chromatography. Bands corresponding to recombinant Pf332-DBL protein are indicated. (c) Mass spectrum profile of Pf332-DBL after size exclusion chromatography to verify purity. One major ion peak at $\mathrm{m} / \mathrm{z} 26935$ can be visualized. (d) Circular dichroism spectrum of Pf332-DBL in the far UV region, demonstrating that the protein is folded and consisting predominantly of $\alpha$-helices, as indicated by a positive band at $195 \mathrm{~nm}$ and two negative bands at 208 and $220 \mathrm{~nm}$.

2.3. Tertiary Structure Modeling of Pf332-DBL. A homology model of 3D7 Pf332-DBL (PF11_0506 amino acids 1-255) was constructed using the HHpred server [16] with default settings. Briefly, the HHpred method is specialized in detecting homology using Hidden Markow Models built from PSI-BLAST profiles and secondary structure. The crystal structure of the EBA-175 F2-domain was used as a template (Protein Data Bank code 1ZRO [17] chain A [18]. The alignment was manually adjusted at positions 180 and 254 to align two cysteines, which are in close proximity, to allow for the modeling of two disulphide bridges conserved in PfEBA175 and Pk $\alpha$-DBP. MODELLER 9v3 [19] and the MPItoolkit were used to create the model. Structure visualization was made in PyMol [21]. The model was validated by using the PROCHECK program [22], available in the SWISSMODEL Workspace [23] and Verify3D, available in the HHpred server toolkit [16].

\subsection{Plasma Samples}

Plasma Samples from Adult Donors. In Uganda, plasma samples were collected from women over 18 years, taking part of a cross-sectional investigation in 2006 on malaria infection in pregnancy at Mulago Hospital, Uganda's national referral hospital in Kampala [24]. Ethical permissions have been obtained both in Sweden and Uganda (permit number 04533/2 and MV922). In Mali, plasma samples were collected at the end of the malaria transmission season in November 2001 in the malaria mesoendemic Mopti area [25]. Adult donors belonged to different sympatric ethnic groups (Fulani and Dogon) living 300-1000 m apart. In Burkina Faso, plasma samples were collected during the peak of malaria transmission season in September and October 1999 in a malaria holoendemic area outside Ouagadougou, the capital of Burkina Faso [25]. Adult donors belonged to either the Fulani group or the Mossi group. Studies in both Mali and Burkina Faso have shown that the Fulani tribe is less parasitized, has higher levels of antimalaria antibodies, and suffers from fewer malaria attacks compared to the Mossi and Dogon tribes despite the same level of malaria transmission [26-28]. Ethical permissions have been obtained both in Sweden and Mali (permit number 03-536 and 1866/FMPOS) and Burkina Faso (permit number 98086 and 9800839/MS/SG/DEP).

Plasma Samples from Children. Plasma samples were collected in 2002 from children under the age of five with an ongoing $P$. falciparum infection in Apac, a malaria 
holoendemic area [29] $250 \mathrm{~km}$ north of Kampala, Uganda [30]. Children were identified with mild or severe malaria according to WHO guidelines [31] and the modified Blantyre scale [32]. Ethical permissions for the study have been obtained both in Sweden and Uganda (permit number 03/095 and MV717).

2.5. Enzyme Linked-Immunosorbent Assay (ELISA). Antibody responses were measured by enzyme linked-immunosorbent assay (ELISA). Maxisorp plates (Nunc, Roskilde, Denmark) were coated overnight at $4^{\circ} \mathrm{C}$ with antigens dissolved in $15 \mathrm{mM} \mathrm{Na}_{2} \mathrm{CO}_{3}$ and $35 \mathrm{mM} \mathrm{NaHCO}_{3}$ (pH 9.6) at a concentration of $10 \mu \mathrm{g} / \mathrm{mL}$. The plates were blocked with $1 \%$ bovine serum albumin (BSA) in PBS containing $0.1 \%$ Tween 20 (PBS-T) for $1 \mathrm{~h}$ at room temperature and subsequently washed three times with PBS-T. All plasma samples were titrated six-fold by serial dilutions in PBS-T to determine concentration dependency, added in duplicates and allowed to bind for $1 \mathrm{~h}$ at $37^{\circ} \mathrm{C}$. The plates were washed and bound immunoglobulin $\mathrm{G}(\mathrm{IgG})$ was detected by incubation for $1 \mathrm{~h}$ at $37^{\circ} \mathrm{C}$ with alkaline phosphataseconjugated goat anti-human IgG (Sigma, St. Louis, MO, USA) diluted 1:1000 in PBS-T. The plates were washed and the assay developed by adding $p$-nitrophenyl phosphate (Sigma) as a substrate for $15 \mathrm{~min}$. The optical density (OD) was read at $405 \mathrm{~nm}$ in an ELISA plate reader (Multiskan EX Version 1.0, Labsystems, Stockholm, Sweden). On each plate, a pool of plasma from 11 nonmalaria exposed Swedish donors was assayed as well as control wells without plasma (background level). Cutoff threshold for seropositivity was determined as the mean $\mathrm{OD}_{405}$ value plus three standard deviations from the 11 Swedish donors. Amount of IgG was expressed as OD ratio and calculated as follows: (OD sample - OD background)/(OD Swedish nonimmune pool - OD background). The presented results and data used for statistical analyses were obtained with plasma samples diluted $1: 500$.

2.6. Affinity-Purification of Pf332-DBL Antibodies from Patient Plasma. The DBL domain of Pf332 was coupled to $\mathrm{CNBr}$-activated sepharose (GE Healthcare) according to manufacturer's instructions and the coupled medium slurry was thereafter degassed and packed into an empty polystyrene column (Thermo Fisher Scientific Inc., Rockford, IL, USA). For purification, $20 \mathrm{~mL}$ of plasma from a pool of adult donors (reactive to Pf332-DBL in ELISAassays) were diluted 1:4 in degassed PBS, sterile filtered, and loaded onto the column and allowed to pass three times. The column was washed extensively with PBS and antibodies were eluted with $150 \mathrm{mM}$ glycine $(\mathrm{pH} 2.5)$ in $10 \times 1 \mathrm{~mL}$ fractions and immediately neutralized with $100 \mu \mathrm{L} 1 \mathrm{M}$ Tris buffer ( $\mathrm{pH}$ 8.6). Antibody concentration was determined spectrometrically, using the molar extinction coefficient of $\varepsilon_{280}=210000 \mathrm{M}^{-1} \mathrm{~cm}^{-1}$ for immunoglobulins. Fractions containing antibodies were pooled, dialyzed against PBS, and finally concentrated using Centriprep centrifugal filter units (Millipore, Billerica, MA, USA).
2.7. Peptide Array. Slides with P. falciparum peptide epitopes were manufactured by JPT (Berlin Germany) and consisted of three identical subarrays. Each subarray had a set of 52 overlapping peptides of 15 amino acids shifted by four residues covering 3D7 Pf332-DBL (PF11_0506, spanning amino acids 1-219) and 148 overlapping peptides covering 3D7 PfEBA-175 (MAL7P1.176, spanning amino acids 145747). The peptides were bound chemoselectively to the microarray surface by coupling of an active amine (from the peptide) to an epoxy-group (from the slide surface). The slides were hybridized with $5 \mu \mathrm{g} / \mathrm{mL}$ human IgG or human affinity-purified Pf332 antibodies diluted in PBS containing $3 \%$ fetal calf serum and $0.5 \%$ Tween 20 (PBS-T) in a humid chamber for $16 \mathrm{~h}$ at $4^{\circ} \mathrm{C}$. Next, the microarray slides were washed five times: two times with PBS-T and three times with distilled water. The slides were subsequently incubated with a secondary Cy5-coupled goat anti-human antibody (1:500; Jackson ImmunoResearch, Suffolk, United Kingdom) for $2 \mathrm{~h}$ at room temperature in a humid chamber, followed by washing steps. The slides were scanned in a GenePix 4000B microarray scanner (Axon Instruments Inc., Foster City, CA, USA) at a wavelength of $635 \mathrm{~nm}$ and images were saved in TIFF and JPG formats. Image analysis was performed utilizing the circular feature alignment of the GenePix Pro 6.0 software (Axon Instruments Inc.) and the GenePix Array List (GAL) files supplied by JPT. To measure antibody response, the mean fluorescence intensity given by the difference between the foreground and the local background of the circular features were used. Data presented represent the average of the three subarrays.

2.8. Parasite Cultures and Kinetic Studies on Synchronous Parasite Populations. P. falciparum clone FCR3S1.2 was maintained in continuous culture according to standard procedures [33]. Briefly, parasites were cultivated at a 5\% hematocrit in malaria culture medium supplemented with $10 \%$ Swedish nonimmune $\mathrm{A}^{+}$serum. Parasites were synchronized with sorbitol treatment and synchronous parasites were harvested at different time-points for indirect immunofluorescence assays and Western blot.

2.9. Indirect Immunofluorescence Assay for Detection of Pf332. For immunofluorescence assays, monolayers of synchronous FCR3S1.2 infected RBC (iRBC) were prepared as previously described. Monolayers were blocked for $1 \mathrm{~h}$ with $3 \%$ BSA in PBS and subsequently incubated for $1 \mathrm{~h}$ with affinitypurified human anti-Pf332-DBL $(1: 2000)$, polyclonal rabbit anti-Pf332-EB200 (1:500), polyclonal rabbit anti-MAHRP1 ( $1: 10$, kind gift from Hans-Peter Beck), monoclonal mouse anti-Pf332-DBL $(1: 800)$, or polyclonal rabbit anti-EBA 175 RVI ( $1: 1600$, kind gift from John H. Adams), all diluted in blocking buffer. Slides were thereafter washed three times with PBS and primary antibodies were detected by incubation for $1 \mathrm{~h}$ with secondary ALEXA-Fluor 594conjugated anti-human IgG, ALEXA-Fluor 488-conjugated anti-rabbit IgG or ALEXA-Fluor 594-conjugated anti-mouse IgG (dilution 1: 1000; Invitrogen, Carlsbad, CA, USA). To 
avoid photobleaching, cells were mounted with Vectashield (Vector Laboratories, Burlingame, CA, USA) containing $1.5 \mu \mathrm{g} / \mathrm{mL} 4^{\prime}$,6-diamidino-2-phenylindole (DAPI) to stain for parasite DNA. All incubations were performed at room temperature in a humid chamber. Cells were viewed with a $100 \times$ oil immersion objective in a UV equipped Nikon Eclipse 90i/80i microscope (Nikon Corporation, Japan) and images were captured by a Hamamatsu Orca-ER CCD Digital camera (Hamamatsu Photonics System, Japan) and MicroManager software version 1.3 (developed by Arthur Edelstein, Nico Stuurman and Nenad Amodaj, University of California, San Francisco, USA). All images were finally processed using ImageJ software version 1.43 (National Institutes of Health, Bethesda, USA).

2.10. Immunodetection of Pf332 in Western Blot. FCR3S1.2 trophozoites $24 \mathrm{~h}$ after invasion (p.i.) were enriched by magnetic cell sorting (Miltenyi Biotec, Bergisch Gladbach, Germany) and returned into culture to reach approximately 36$40 \mathrm{~h}$ p.i. Samples were lysed in SDS-loading buffer, separated on a $6 \%$ SDS-PAGE $\left(2.5 \times 10^{5} \mathrm{iRBC} /\right.$ lane $)$ and transferred onto nitrocellulose membranes (Bio-Rad, Hercules, CA, USA). Membranes were probed with affinity-purified human anti-Pf332-DBL $(1: 3000)$, anti-EB200 $(1: 1000)$, and monoclonal anti-Pf332-DBL $(1: 100)$. Detection by enhanced chemiluminescence (ECL plus Western blotting detection reagents, GE Healthcare) was performed after a secondary probe of HRP-coupled anti-human IgG, antirabbit IgG, or anti-mouse IgG $(1: 5000$, GE Healthcare) were added.

2.11. Statistical Analyses. Statistical analyses were performed with Prism version 5.0 (Graphpad Software, San Diego, USA). Chi-square test was used when comparing the seroprevalence. Student's paired $t$-test or Wilcoxon signed ranked test (when samples were not normally distributed) was used when comparing antibody levels within the same group. Student's unpaired $t$-test or Mann-Whitney test (when samples were not normally distributed) was used when comparing antibody levels between different groups.

\section{Results}

3.1. Purification and Characterization of Pf332-DBL Recombinant Protein. The DBL domain of Pf332 was expressed in E. coli as a His-tagged recombinant protein and purified by $\mathrm{Ni}$-affinity chromatography, followed by size exclusion chromatography. A band migrating at approximately $27 \mathrm{kDa}$ could be visualized on the Coomassie-stained SDSpolyacrylamide gel from the soluble E. coli lysate, as well as from the eluted fractions after affinity purification and size exclusion chromatography (Figure 1(b)). The protein eluted from the size exclusion column at an elution volume of $90 \mathrm{~mL}$, which corresponds to the size of a monomer. To verify the molecular mass of the purified protein, analysis by mass spectrometry was conducted. The mass spectra showed one major ion at $\mathrm{m} / \mathrm{z} 26935$, which corresponds to the theoretical weight of Pf332-DBL (Figure 1(c)).
To evaluate the folding state and secondary structure content of the recombinant protein, $\mathrm{CD}$ spectra were measured in the far UV region. The spectra indicate that the protein is folded, and the shape of the spectra, with a positive band at $195 \mathrm{~nm}$ and two negative bands at 208 and $220 \mathrm{~nm}$, respectively, is typical for proteins with a high $\alpha$ helical content (Figure 1(d)) [34].

3.2. Homology Modeling of the Structure of the Pf 332 DBL-Domain. Crystal structures of two different EBL-DBL domains have been determined, namely, the tandem DBL domains of P. falciparum EBA-175 (F1 and F2) [18] and the single DBL domain of $P$. knowlesi Duffy-binding protein (Pk $\alpha$-DBL) [35]. Using the crystal structure of EBA-175 F2 as a template (PDB code 1ZRO [18]), a three-dimensional (3D) model of Pf332-DBL was constructed by comparative modeling on the basis of the 3D7 sequence (PF11_0506, amino acids 1-255) in Modeller 9v3 [19]. The HHpred probability score for EBA-175 F2 to be used as a template for Pf332-DBL modeling was $100 \%$, although the sequence identity between the two domains was only 29\% (Figure 2(a)). The model was validated by using the PROCHECK program [22] and Verify3D [16]. The PROCHECK Ramachandran plot showed that $90.1 \%$ of the residues in the model were in the core region, $9.1 \%$ in the allowed region, $0.4 \%$ in the generously allowed region, and $0.4 \%$ in the disallowed region, proving that the 3D model was of good quality. Analysis using Verify3D resulted in a similar conclusion (score $>0.1$ ).

The homology model of Pf332-DBL consists predominantly of $\alpha$-helices, which is in accordance with our CDspectrum (Figure 2(b)). The model further adopts a basic fold similar to both the DBL domains of EBA-175 [18] and $\mathrm{Pk} \alpha$-DBL [35]. Of the twelve cysteine residues present in Pf332-DBL, ten correspond in location to cysteines in EBA175 F2 and eight to cysteines shared between EBA-175 F1, F2, and Pk $\alpha$-DBL (Figure 2(a)). Five disulphide bridges were further identified in the model and four of them were found to be conserved in EBA-175 F1, F2, and Pk $\alpha$-DBP whilst the fifth was only found in EBA-175 F2. Since our homology model of Pf332-DBL is structurally similar to EBL-DBLs, we used the Singh et al. nomenclature [35] and divided the Pf332-DBL domain into three subdomains (Figure 2(c)). In the model, a random-coil stretch of residues constitutes subdomain 1. All DBL domains crystallized to date have four invariant cysteine residues forming two conserved disulphide bridges in this subdomain. Pf332-DBL, however, lacking two of these cysteines (Cys 1 and 4; Figure 2(a)), only has one disulphide bridge in this region (Cys 2-3; Figure 2(c)). Subdomain 2 of the Pf332 model contains four $\alpha$-helices connected by loops. One conserved disulphide bridge could be identified in the subdomain (Cys 5-6; Figure 2(c)) as well as two unpaired cysteines (Cys $5^{*}$ and $6^{*}$ ), which were found to be unique for Pf332-DBL (Figure 2(a)). Subdomain 3 has one short stretch of $\alpha$-helices but the subdomain is dominated by its two long antiparallel $\alpha$-helices that are connected by a loop. The third subdomain is predicted to be held together by three disulphide bridges, two of which are conserved among all DBL domains studied here (Cys 8- 


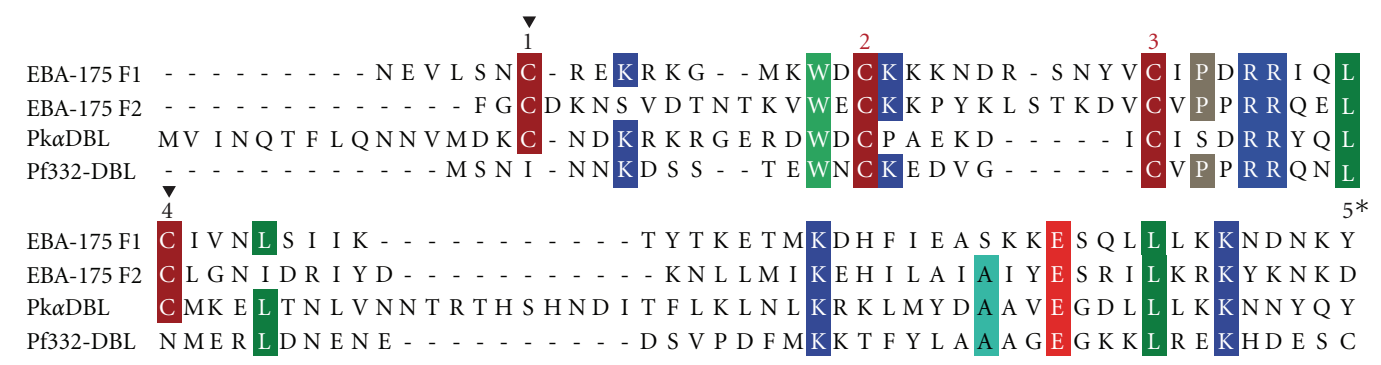

EBA-175 F1 NS K F C N D L KN S F L DYG H L A MGNDMD F GGY S T K A ENK I Q E VFKGAHGE I S E

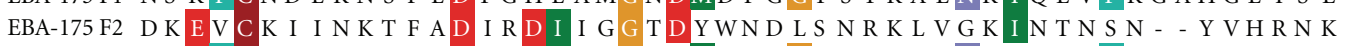
Pk $\alpha$ DBL NKE FCKD I RWG L GDFGD I I MG T NME G I GY S QVVENN LR S I F G . . . . . T D Pf332-DBL D - E F CDAWN R L ADYKD I F Q G K DWN D GKY G E A KH I K N A F G . . . . .

EBA-175 F1 HK IKN F R K KWWNEFR E K LWEAML S EHKNN I N … - - C KN - - I P Q EELQ I EBA-175 F2 QNDKL F R D EWWKVI K K DVWNV I SWVFKD- . . - K T VCKED - D I E N I P Q F Pk $\alpha$ DBL E K A K QDR K QWWNESKEH I WR AMM F S L R S R L K E K F VW I C K K D V T L K VE P Q I

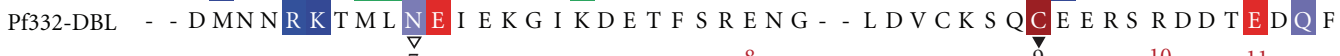

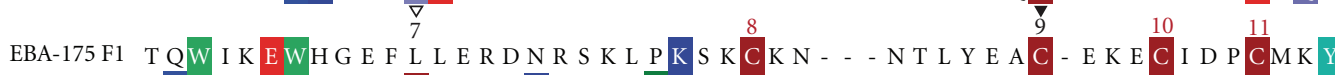
EBA-175 F2 F RWF S EWGDDYCQDKTKM I E T L KVECK E . . . . K P CEDDNCKR KCN S Y Pk $\alpha$ DBL YR I R E WGR D Y MS E L P K E Q GK L N E KCA S K L Y Y NNMA I CML P L CHD A C K S Y Pf332-DBL L R F F A EWE E E F CDGL KKHE E Q L K S C T KD......... I NCD I KCS N F EBA-175 F1 R DW I I R S K F EWH T L SK E Y - . . . - E T QKV P K E NA E NY L I K I S E N KN - . EBA-175 F2 K EW IS KKK E E Y N K QAKQYQEYQKGNNYKMY S E F K S I K P E V Y L KKY S E K C S Pk $\alpha$ DBL DQW I T R K K QWDVL T K F S V K - - K T QK I G T E N I A T A Y D I L K Q E L N G F K Pf332-DBL K DWL E TKKDEY D I QSR V F ....... E KKY A N D N S K H L N Y L K E G MNKC K

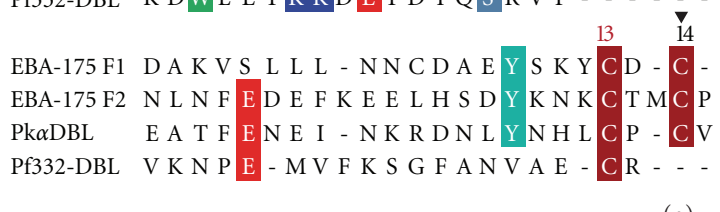

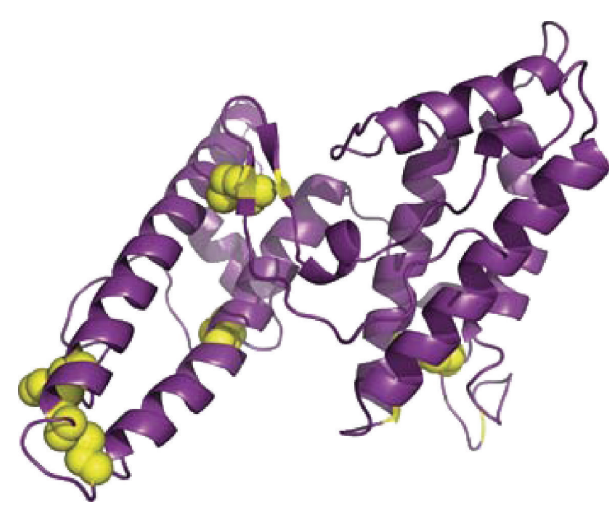

(b)

(a)

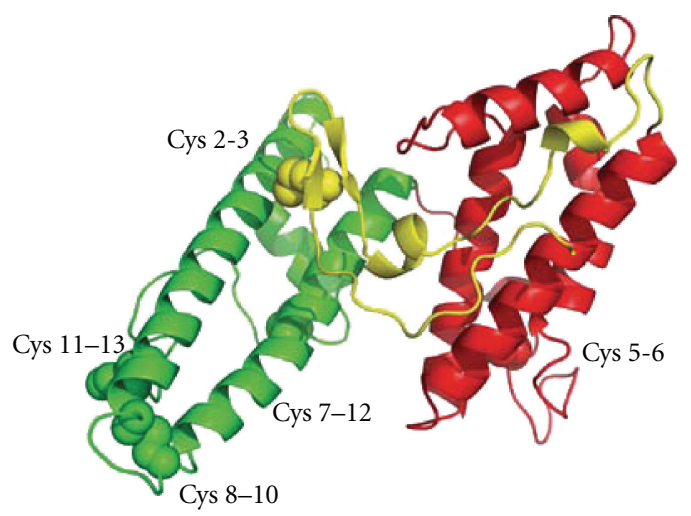

(c)

Figure 2: Homology model of Pf332-DBL created using EBA-175 as a template. (a) Sequence alignment of PfEBA-175 F1 and F2 (amino acids 152-426 and 460-743, resp.), Pk $\alpha$-DBL (amino acids 15-317) and Pf332-DBL (amino acids 1-255). The sequence identity between Pf332 and EBA-175 F1, F2, and Pk $\alpha$-DBL is 26\%, 29\%, and 24\%, respectively. Cysteines are numbered according to the occurrence in the EBA-175 F2 template. Of the twelve cysteines encoded by Pf332-DBL, eight are conserved between all DBL domains investigated here (Cys numbering displayed in red), two are conserved with only EBA-175 F2 (marked with white arrowheads) whilst the remaining two are unique for Pf332-DBL (numbering marked with an asterisk). Cysteines that are missing in the Pf332-DBL domain but that are present in EBA-175 F1, F2, and Pk $\alpha$-DBL are marked with black arrowheads. (b) Homology model of Pf332-DBL (PF11_0506 amino acids 1-255) created using EBA-175 F2 (PDB code 1ZRO) as a template. Cysteines are shown in yellow, and residues predicted to be involved in disulphide bridges are shown as spheres. (c) The predicted Pf332-DBL model can be subdivided into three subdomains; subdomain 1 (yellow), subdomain 2 (red), and subdomain 3 (green). Subdomains 1 and 2 have one disulphide bridge each (Cys 2-3 and Cys 5-6, resp.) whilst subdomain 3 has three (Cys 7-12, Cys 8-10, and Cys 11-13). For Pf332-DBL, Cys $2=$ Cys residue 15, Cys $3=$ residue 21, Cys 5* = residue 69, Cys $5=$ residue 73, Cys $6^{*}=$ residue 140 , Cys $6=$ residue 144 , Cys $7=$ residue 168 , Cys $8=$ residue 181 , Cys $10=$ residue 187 , Cys $11=$ residue 191 , Cys $12=$ residue 235, and Cys $13=$ residue 254 . 
10 and Cys 11-13) and one that is shared with only EBA175 F2 (Cys 7-12; Figure 2(c)). The third subdomain further lacks two cysteines that are present in all other DBL domains studied here (Cys 9 and 14; Figure 2(a)).

3.3. Seroprevalence and Magnitude of Naturally Acquired Antibodies to Pf332-DBL and PfEMP1-DBL1 $\alpha$. In order to examine whether antibodies towards the DBL domain of Pf332 were elicited during natural infection with $P$. falciparum, the recombinant Pf332-DBL protein was examined together with a DBL1 $\alpha$-domain from the variant surface antigen PfEMP1 in ELISA assays. Seroprevalence and antibody levels were analyzed in plasma samples collected from semi-immune adults in Kampala (Uganda) and children with either mild or severe malaria in Apac (Uganda). We further analyzed plasma samples from asymptomatic individuals with different susceptibility to malaria, belonging to sympatric ethnic groups in Burkina Faso (the Fulani and the Mossi tribe) and Mali (the Fulani and the Dogon tribe). As summarized in Table 1, the seroprevalence of antibodies directed against Pf332-DBL and PfEMP1-DBL1 $\alpha$ was consistently high in the distinct malaria endemic regions studied here (60-98\% and 50-87\%, resp.), demonstrating the immunogenicity of the two antigens.

In Kampala, over $60 \%$ of the individuals were seropositive for Pf332-DBL and PfEMP1-DBL1 $\alpha$ (Table 1) and they had acquired comparable levels of antigen-specific antibodies (median OD ratio of immune plasma/Swedish nonimmune plasma were 2.7 and 3.7, resp.; Figure 3(a)). In the highly endemic area of Apac, over $71 \%$ of the children with mild malaria and $84 \%$ of the children with severe malaria were seropositive for the two antigens already at a very young age ( 7 months to 4 years; Table 1$)$. Moreover, all children had acquired comparable levels of antigenspecific antibodies, regardless of whether they had mild or severe malaria (median OD ratio ranging from 4.0 to 4.8 ; Figure 3(b)).

Adults living in Burkina Faso had the highest Pf332-DBL seroprevalence with over $95 \%$ positive individuals in both the Fulani and the Mossi tribe (Table 1). Furthermore, individuals in both tribes had acquired similarly high levels of Pf332DBL specific antibodies (median OD ratio 7.4 in the Fulani and 8.1 in the Mossi; Figure 3(c)). The seroprevalence of antibodies directed against PfEMP1-DBL1 $\alpha$ differed between the two ethnic groups and a significantly higher proportion of individuals of the Mossi tribe were found to be seropositive ( $73 \%$ of the Mossi compared to $52 \%$ of the Fulani, $P<0.05$ ). The amount of antibodies specific for PfEMP1-DBL1 $\alpha$ was, however, similar in both tribes (median OD ratio 2.6 in the Fulani and 3.4 in the Mossi; Figure 3(c)). When comparing antibody levels within each ethnic group, levels of antibodies directed against Pf332-DBL were found to be significantly higher than levels of PfEMP1-DBL1 $\alpha$ antibodies in both the Fulani and the Mossi tribe $(P<0.001)$.

In Mali, the seroprevalence of Pf332-DBL antibodies was over $93 \%$ in both the Fulani and the Dogon group (Table 1) and $\operatorname{IgG}$ levels were similar and very high (median OD ratio 7.6 in the Fulani and 7.2 in the Dogon; Figure 3(d)).
Opposite to what was observed in Burkina Faso, more Fulani than Dogon were seropositive for antibodies directed against PfEMP1-DBL1 $\alpha$ ( $71 \%$ of the Fulani compared to $50 \%$ of the Dogon), although this difference was not significant (Table 1). The Fulani tribe, which is less susceptible to malaria, had acquired significantly higher $(P<0.01)$ levels of antibodies towards PfEMP1-DBL1 $\alpha$ than their sympatric tribe Dogon (median OD ratio 3.2 in the Fulani and 2.7 in the Dogon, Figure 3(d)). Similar to what was observed in Burkina Faso, levels of Pf332-DBL antibodies were found to be significantly higher than levels of PfEMP1-DBL1 $\alpha$ antibodies in both the Fulani and the Dogon tribe $(P<$ $0.001)$.

\subsection{Peptide Array to Determine Epitope Specificity of Naturally} Acquired Human Pf332-DBL Antibodies. Previous studies on Pf332 have been hampered by the potential cross-reactivity of anti-Pf332 antibodies with other repetitive antigens [36]. In an effort to evaluate the fine specificity of naturally acquired human antibodies to Pf332-DBL, we affinitypurified human antibodies specific for the Pf332 DBL domain from adult plasma displaying reactivity towards the region in ELISA-assays. These antibodies were subsequently probed on a peptide array of 52 overlapping 15-mers (spanning amino acids 1-219 of Pf332). The Pf332-DBL antibodies recognized two overlapping peptides, having the sequence KKDEYIDIQSRV in common (Figure 4(a)). When mapped on the 3D model of Pf332-DBL, the epitope was found to be located on one of the two antiparallel $\alpha$-helices in subdomain 3 (Figure 4(b)). To rule out the possibility of cross-reactivity with epitopes in the DBL domains of the EBL family of invasion proteins, we also probed a peptide array of 15-mers spanning amino acids 145-747 covering both the F1 and F2-domain of EBA-175, with the human antibodies. The anti-Pf332 antibodies did not bind to any of the EBA175 peptides, indicating that they do not cross-react with linear epitopes of the EBL-DBLs (see Supplementary Figure1 available online at doi: 10.4061/2011/671439).

\subsection{Specificity Studies of Native Pf332 Protein Expression.} In order to analyze the ability of the naturally acquired antibodies to detect native Pf332 protein, we performed immunofluorescence assays with the affinity-purified human anti-Pf332-DBL antibodies on air-dried monolayers of RBC infected with the FCR3S1.2 parasite strain. At 28-32 h p.i., the human anti-Pf332-DBL antibody reacted with Maurer's cleft like structures in the iRBC cytosol (Figure 5(a)). The antibody colocalized with an antibody specific for the Pf332 repeat region EB200, confirming the specificity of the affinity-purified human anti-Pf332-DBL antibody (Figure 5(a)).

To further investigate antibody specificity, we carried out immunofluorescence assays using a monoclonal antibody against Pf332-DBL and antibodies targeting the Pf332 repeats, Maurer's clefts, and the EBL family of invasion proteins. At 30-34 h p.i., the anti-Pf332-DBL antibody showed colocalization with anti-Pf332-EB200, confirming that the 


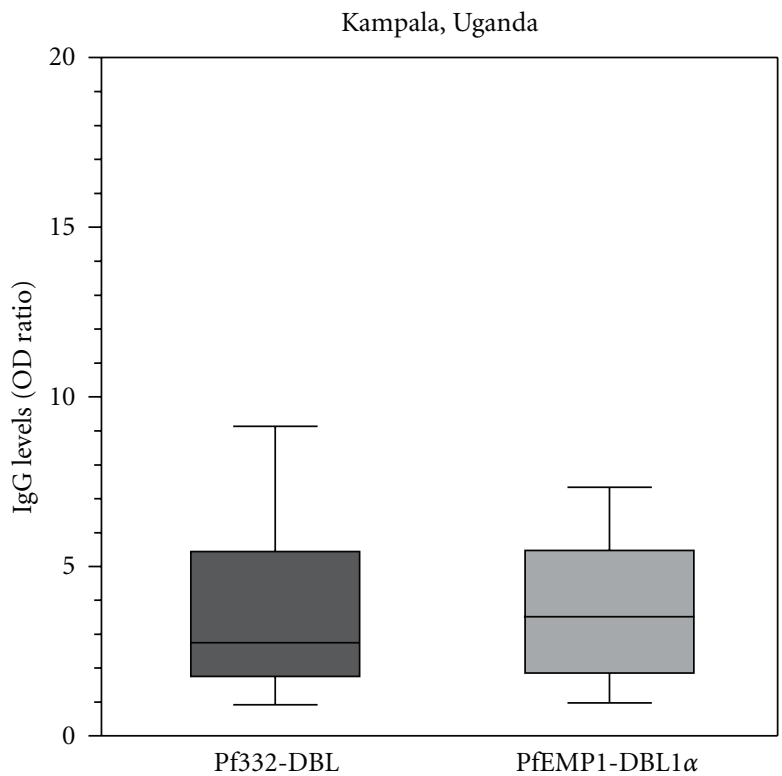

(a)

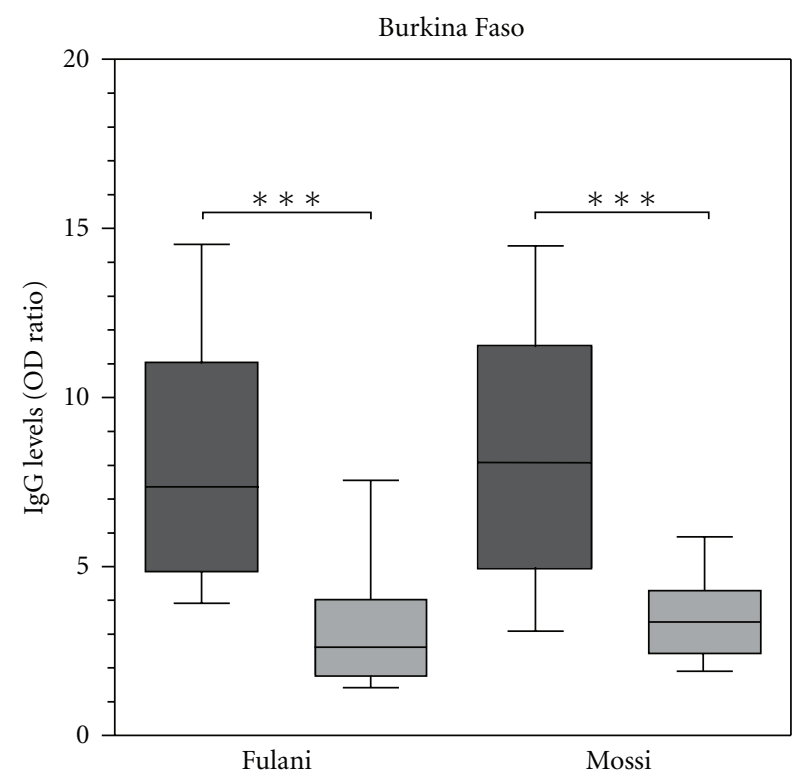

Pf332-DBL

PfEMP1-DBL1 $\alpha$

(c)

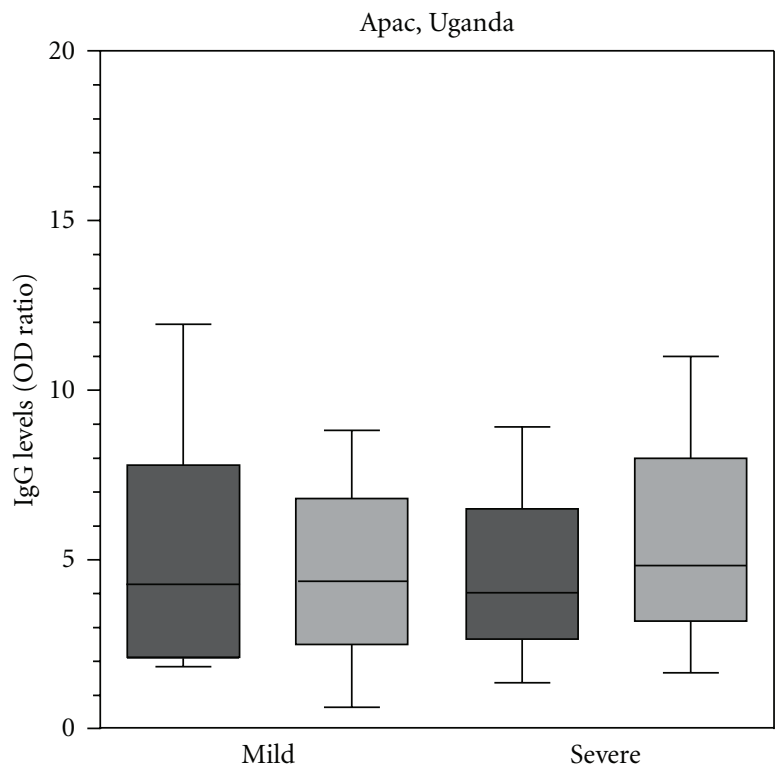

Pf332-DBL

PfEMP1-DBL1 $\alpha$

(b)

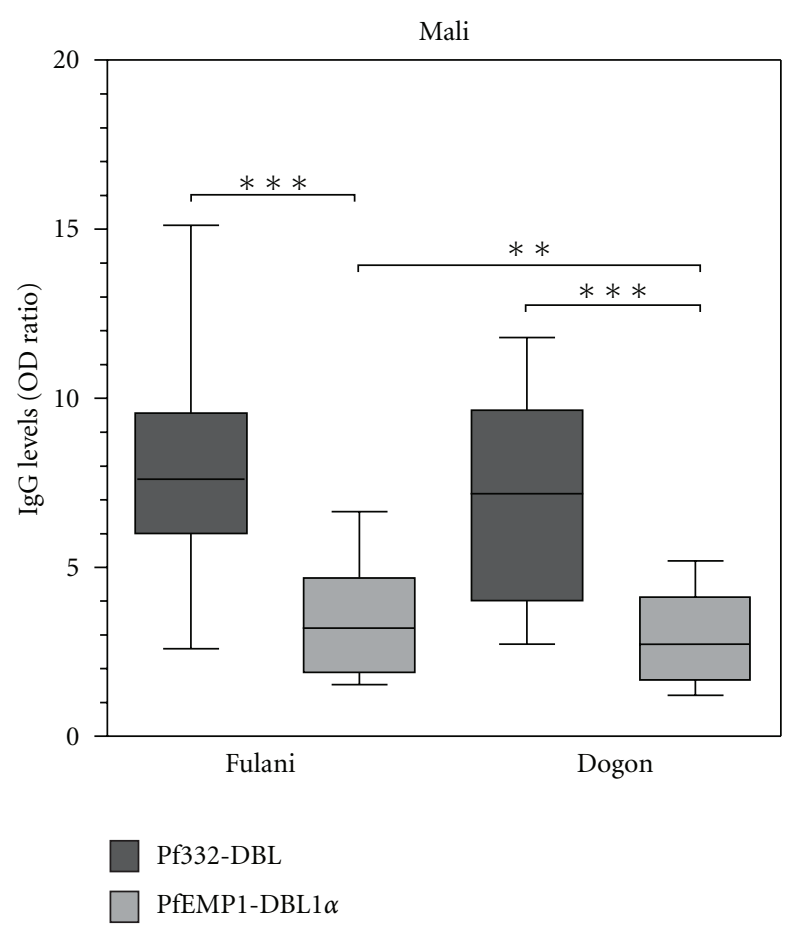

(d)

Figure 3: IgG reactivity to Pf332-DBL and PfEMP1-DBL1 $\alpha$ in children and asymptomatic adults. Antibodies specific for Pf332-DBL and PfEMP1-DBL1 $\alpha$ in plasma samples collected from asymptomatic adults in Uganda, Burkina Faso, and Mali as well as from children with mild or severe malaria in Uganda. Plasma samples were analyzed in ELISA at a dilution of $1: 500$. IgG reactivity is expressed as OD ratio and calculated as follows: (OD sample - OD background)/(OD Swedish nonimmune donor pool - OD background). (a) Adults in Kampala, Uganda; (b) children with mild or severe malaria in Apac, Uganda; (c) adults in Burkina Faso and (d) adults in Mali. Box plots show medians with 25th and 75th percentiles and whiskers for 10th and 90th percentiles. $* *$ and $* * *$ indicate significant differences between groups $(P$ $<0.01$ and $P<0.001$, resp.). 
TABLE 1: Prevalence of seropositivity for antibodies against Pf332-DBL and PfEMP1-DBL1 $\alpha$.

\begin{tabular}{|c|c|c|c|c|}
\hline Countries (ethnic group) & $n$ & $\begin{array}{c}\text { Pf332-DBL } \\
\text { No. }(\%) \text { of responders }{ }^{1}\end{array}$ & $\begin{array}{c}\text { PfEMP1-DBL1 } \alpha \\
\text { No. }(\%) \text { of responders }{ }^{1}\end{array}$ & $P$ value ${ }^{2}$ \\
\hline Uganda, Kampala & 120 & $72(60.0)$ & $82(68.3)$ & ns \\
\hline Uganda, Apac, mild malaria & 39 & $29(74.4)$ & $28(71.8)$ & ns \\
\hline Uganda, Apac, severe malaria & 31 & $26(83.9)$ & $27(87.1)$ & ns \\
\hline Burkina Faso (Fulani) & 60 & $57(95.0)$ & $31(51.7)$ & $<0.0001$ \\
\hline Burkina Faso (Mossi) & 48 & $47(97.9)$ & $35(72.9)$ & 0.0008 \\
\hline Mali (Fulani) & 28 & $26(92.9)$ & $20(71.4)$ & ns \\
\hline Mali (Dogon) & 32 & $30(93.8)$ & $16(50.0)$ & 0.0002 \\
\hline
\end{tabular}

${ }^{1}$ Cutoff threshold for seropositivity was determined as the mean $\mathrm{OD}_{405}$ value plus $3 \mathrm{SD}$ of a pool of nonimmune Swedish donors.

${ }^{2} P$ values by Chi-square test at a $<0.05$ significance level ( $\mathrm{ns}=$ not significant).

monoclonal anti-DBL antibody was able to detect native Pf332 (Figure 5(b), upper panel). Anti-Pf332-DBL further colocalized with MAHRP1, a known Maurer's clefts marker in P. falciparum [37], which is in accordance with what has previously been described for Pf332 [6, 7] (Figure 5(b), middle panel). At $40 \mathrm{~h}$ p.i., we could detect Pf332 in Maurer's cleft like structures in close proximity to the RBC plasma membrane. These antibodies did not co-localize with antiEBA-175 antibodies, which gave a punctuate fluorescence pattern typical for antigens localized within the merozoite apical complex (Figure 5(b), bottom panel), demonstrating the lack of cross-reactivity between the two antigens.

To confirm the specificity of the anti-Pf332-DBL antibodies, Pf332 expression was analyzed by Western blot. Pf332 could be detected as a high-molecular-weight band migrating well above the $170 \mathrm{kDa}$ marker in FCR3S1.2 parasite extract, when probing with affinity-purified human anti-Pf332-DBL, anti-EB200, and monoclonal anti-Pf332DBL antibodies (Figure 5(c)). Some smaller bands, which are likely processed protein or proteolytic degradation products of full-length Pf332, were also observed. No reactivity was detected with any of the antibodies in uninfected RBC.

\section{Discussion}

Although Pf332 was identified two decades ago [8-10], the function of this giant protein still remains elusive. A number of possible functions have, however, been described for Pf332 and among them are involvement in rigidity modifications of $\mathrm{iRBC}[38,39]$, indirect adhesion to uninfected $\mathrm{RBC}$ by assisting in transportation of PfEMP1 to the iRBC surface [38], DBL-mediated adhesion to uninfected RBC [4], and involvement in parasite growth [4, 40-43]. Pf332 is present in all isolates investigated $[4,10]$ indicating that the molecule is of importance for the parasite. However, Pf332 does not seem to be essential as parasites with a disrupted Pf332 gene are capable of replicating normally in vitro although the iRBC appears to be more rigid [38, 39].

To date, two different EBL-DBL crystal structures are known; the tandem DBL domains of P. falciparum EBA175 (referred to as F1 and F2) and the single DBL domain of P. knowlesi Duffy-binding protein ( $\mathrm{Pk} \alpha-\mathrm{DBL})$. Despite low sequence identity, all three DBL domains have highly similar structures. All three share several invariant cysteine residues, which are involved in conserved disulphide bridges, demonstrating the importance of the cysteines in maintaining the DBL fold. It is interesting that Pf332-DBL, having only approximately $24-29 \%$ sequence identity with the DBL domains of Pk $\alpha$-DBL and EBA-175 but having the majority of its cysteine residues at conserved locations, shares this basic DBL-fold according to our model (Figure 2). However, there are some differences between the Pf332 model and the crystallized DBL domains. First, Pf332-DBL lacks two cysteine residues in subdomain 1, which form a conserved disulphide bridge in all DBL domains crystallized to date. This disulphide bridge holds two loops together in $\mathrm{Pk} \alpha$ DBL and EBA-175 and the lack of a disulphide bridge in our model is likely to allow more flexibility in this region of Pf332-DBL, which could be of functional importance. Second, Pf332-DBL has two unique cysteines in subdomain 2 , which are not involved in forming a disulphide bridge in our model, but have been predicted to do so by others [39]. The addition of a unique disulphide bridge further allows for speculations about structural differences in the DBL domain of Pf332 compared to the DBL domains of the EBL members. Third, Pf332-DBL is missing two cysteines in subdomain 3, which form a disulphide bridge in all three DBL domain crystal structures analyzed here. It seems clear that the overall structure of Pf332-DBL is similar to the DBL domains of the EBL family. However, the structural differences observed imply that Pf332-DBL could have a different dynamical behavior, leading to differences in its receptor specificity or binding preferences.

It is intriguing why Pf332 has a DBL domain homologous to the EBL family, although it lacks the classic EBL gene structure and the typical EBL expression profile. In contrast to the EBL members, Pf332 contains predominantly degenerate repeats, which in previous studies has been shown to bind actin and as a result, modulate the rigidity of the iRBC $[38,39,44]$. However, a clear function for the DBL domain has not been described. The F1-domain of EBA-175 is related to the single DBL domain of $P$. vivax and $P$. knowlesi $[3,18]$, and the domain is common to all EBLs [45]. In contrast, the F2-domain of EBA-175 has so far only been found in $P$. falciparum and $P$. reichenowi and the domain is suggested to be the progenitor of var DBL [45]. It is therefore interesting 
Pf332-DBL

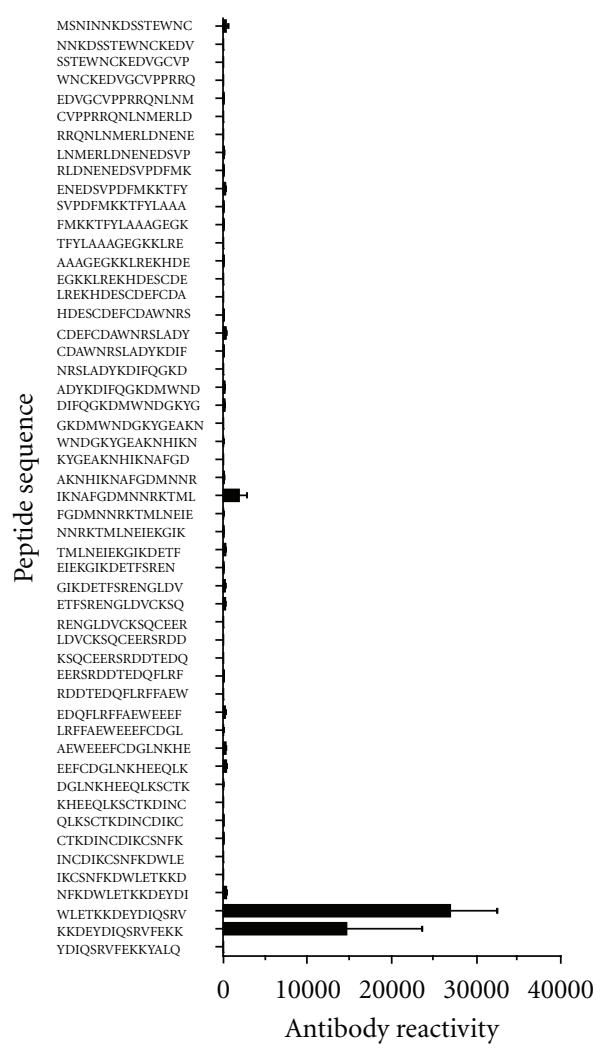

(a)

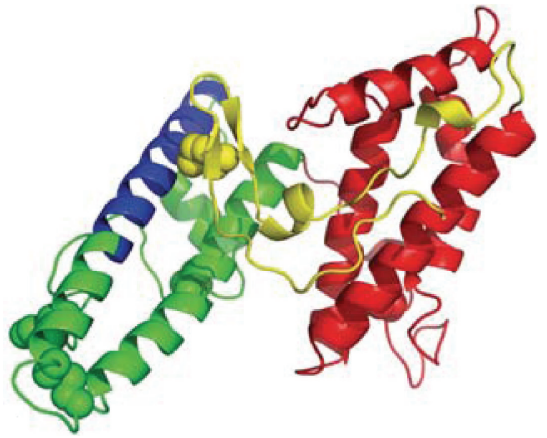

(b)

FIGURE 4: Fine epitope mapping of naturally acquired human anti-Pf332-DBL antibodies on a peptide array. (a) The fine specificity of human affinity-purified anti-Pf332-DBL antibodies was analyzed by a peptide microarray with 52 overlapping peptides of 15 amino acids shifted by four residues covering 3D7 Pf332-DBL (amino acids 1-219). Two overlapping 15 mers having the peptide sequence KKDEYIDIQSRV in common were recognized by the human antibodies. Nonimmune IgG gave a reactivity lower than $250 \pm 200$, and is therefore not displayed in the graph. Peptides are ordered from N-terminus (top) to C-terminus (bottom). Bars represent the mean reactivity of three subarrays; error bars indicate standard deviation. (b) Mapping of epitopes recognized by the affinity-purified human anti-Pf332-DBL antibody on the 3D model of Pf332-DBL. Subdomain 1 (yellow), subdomain 2 (red), and subdomain 3 (green). The epitope recognized by the human affinity-purified anti-Pf332-DBL antibodies (blue) is located on one of the two major $\alpha$-helices in subdomain 3 . Disulphide bridges are displayed as spheres.

to note that the DBL domain of Pf332 has more similarities to F2 than to F1 of EBA-175, as the DBL domain of antigen 332 so far only has been identified in $P$. falciparum, although antigen 332 orthologues are present in species such as $P$. berghei and P. yoelii [46].

Our data show that antibodies to Pf332-DBL are frequent in plasma from individuals residing in distinct malaria endemic regions, demonstrating that it is immunogenic. The frequent seropositivity is also in accordance with the domain being conserved and having very few polymorphisms observed [4]. The lack of variation in the DBL domain of Pf332 suggests that the protein is concealed from the immune system and therefore is not under any selective pressure. As a result, it is likely that antibodies towards the antigen are generated upon schizont rupture, at which time the protein is released into the extracellular environment. Previous studies using recombinant protein of the Pf332 regions EB200 and C231 (a C-terminally located region of Pf332, less rich in degenerate-repeats [47]), have reported that children lack antibodies against both parts of the molecule. However, antibodies were frequently found in older individuals, indicating a slow acquisition of Pf332 antibodies [14, 48]. Our results show that children living in Apac, a highly endemic region in Uganda experiencing the highest entomological inoculation rate reported in Africa [29], had developed antibodies to both Pf332-DBL and PfEMP1-DBL already at a very young age. Due to conservation of Pf332-DBL, antibodies should be acquired early in life in nonimmune children and particularly in areas of high malaria endemicity, which is confirmed by our study. It must, however, be taken into consideration that plasma samples were collected from children with an ongoing $P$. falciparum infection where the host immune system had been recently boosted. High levels of antibodies against PfEMP1-DBL1 $\alpha$ were also frequently found among children in the Apac area, although the sequence diversity of this domain is great. This might be a result of cross-reactivity to similar PfEMP1 variants. 

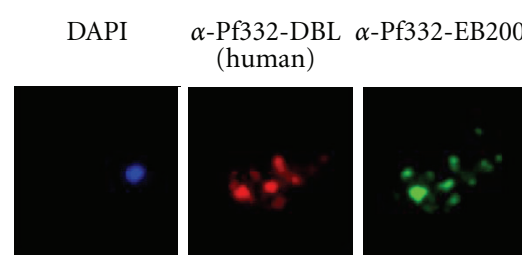

(a)

DAPI

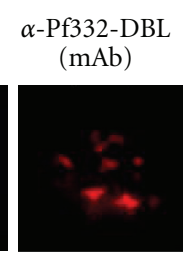

$\alpha$-Pf332-DBL

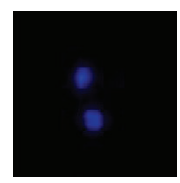

DAPI
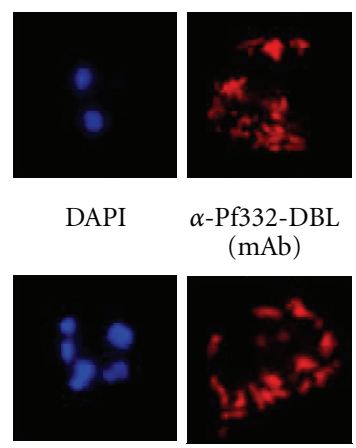

$\alpha$-Pf332-DBL $(\mathrm{mAb})$

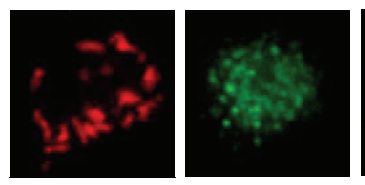

(b)
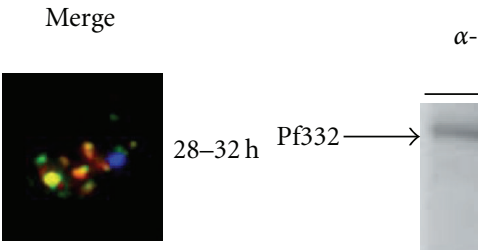

$\alpha$-Pf332-DBL
(human)

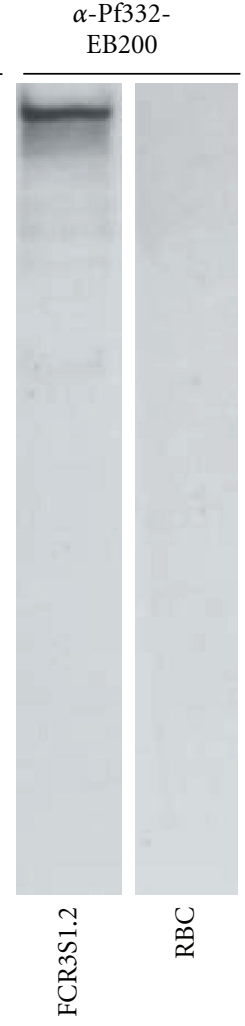

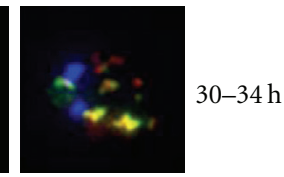

Merge

\footnotetext{
(1)
}

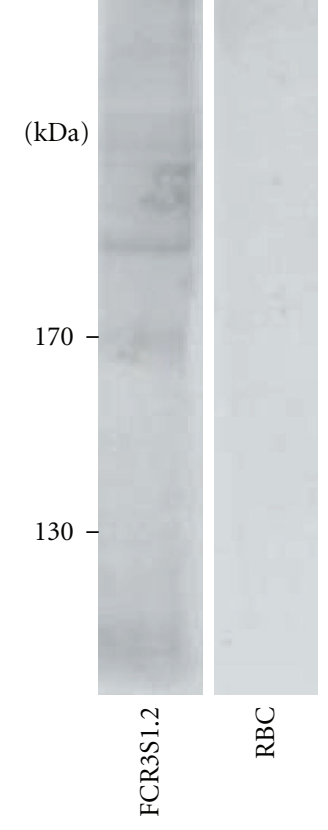

(c)
40-44 h

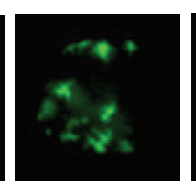

$\alpha$-EBA-175
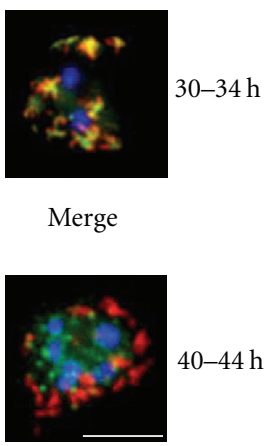

Merge

FIGURE 5: Detection of native Pf332 in immunofluorescence assays and Western blot. Protein expression of Pf332 in FCR3S1.2 infected red blood cells (iRBC). (a) Immunofluorescence analysis on air-dried monolayers of iRBC (28-32 h p.i.) with human affinity-purified antiPf332-DBL antibodies (red) and anti-Pf332-EB200 (green). The parasite was counterstained with DAPI (blue). The antibodies colocalized well and detected Pf332 in Maurer's cleft like structures in the iRBC cytosol. (b) At 30-34 h p.i., a monoclonal antibody against Pf332-DBL (red) showed colocalization with both anti-Pf332-EB200 (green; upper panel) and anti-MAHRP1, a known Maurer's cleft marker (green; middle panel). In schizonts, anti-Pf332-DBL (red) could be detected in Maurer's clefts in close proximity to the RBC plasma membrane. These antibodies did not co-localize with anti-EBA-175 antibodies (green), which gave a punctuate fluorescence pattern typical for antigens localized within the merozoite apical complex. The parasite was counterstained with DAPI (blue). Scale bar indicates $5 \mu \mathrm{m}$. (c) Western blot analysis of Pf332 expression in FCR3S1.2 iRBC (36-40 h p.i.). Membranes were probed with human affinity-purified anti-Pf332-DBL (lane 1 and 2), polyclonal anti-Pf332-EB200 (lane 3 and 4), and monoclonal anti-Pf332-DBL (lane 5 and 6). Bands corresponding to full length Pf332 are indicated. All three antibodies recognized Pf332 migrating well above the $170 \mathrm{kDa}$ marker. None of the antibodies reacted with uninfected RBC.

Antibodies against the DBL domain of Pf332 were highly prevalent among individuals in both Burkina Faso and Mali regardless of their ethnic group and antibodies occurred at very high titers. In contrast, the responder frequency to PfEMP1-DBL1 $\alpha$ differed considerably between the ethnic groups. One explanation might be that although living close to each other geographically and experiencing the same malaria transmission rate, the different tribes had encountered parasites presenting different PfEMP1 molecules on the iRBC surface. Whilst the Pf332 gene is present as a single copy gene in most parasite isolates studied so far [9], the var genes encoding the PfEMP1 molecule are present in approximately 60 copies per genome $[49,50]$. In Mali, the Fulani who are less susceptible to malaria were found to have significantly higher antibody levels to PfEMP1-DBL1 $\alpha$ than their sympatric tribe Dogon. Further, the Fulani were more seropositive, indicating either that they more frequently had encountered parasites expressing this particular or similar subtypes of PfEMP1 to which they had acquired antibodies, or that they more easily develop high levels of antibodies, which could be an explanation for their lower susceptibility to malaria.

Studies on Pf332 have been hampered by the crossreactive nature of the antigen due to its high content of negatively charged repeats, which are present in several malaria antigens. The DBL domain of Pf332 is conserved between different parasite isolates, thus enabling more antigen specific studies. In an effort to look at antibody specificity and to exclude the possibility of cross-reactivity to DBL domains of the EBL family of invasion proteins, we affinitypurified human antibodies using the DBL domain of Pf332 and analyzed them in peptide arrays, immunofluorescence microscopy assays, and Western blotting. From the peptide microarray it was evident that the antibodies only reacted 
with epitopes found in Pf332 and not in DBL domains of the EBL family of invasion proteins, here represented by the tandem DBL domains of EBA-175 (Figure 4 and Supplementary Figure1). This method only takes linear epitopes into consideration; however, it gives a strong indication that there is very little or no cross-reactivity between the DBL domains of Pf332 and the EBL family. The lack of cross-reactivity when using native protein was confirmed by immunofluorescence microscopy and Western blotting (Figure 5), which concludes that antibodies raised against the DBL domain of Pf332 do not cross-react with the DBL domains of the EBL family.

\section{Conclusions}

In summary, the DBL domain represents a conserved region of the Pf332 molecule, with a similar structure to the DBL domains of EBA-175 and Pk $\alpha$-DBP according to our 3D predictions. Antibodies towards the domain are acquired early in life in areas of high endemicity and antibodies are commonly found in semi-immune adults. Moreover, antibodies towards Pf332-DBL are antigen specific and do not display cross-reactivity with DBL-containing EBL proteins. Hence, the DBL domain of Pf332 is expected to greatly facilitate further investigations of Pf332; including its function and potential role in immunity to malaria.

\section{Abbreviations}

DBL: Duffy-binding-like

EBL: Erythrocyte-binding-like

iRBC: Infected red blood cell

PBS-T: Phosphate buffered saline Tween

BSA: Bovine serum albumin

CD: Circular dichroism

PDB: Protein data bank

OD: Optical density.

\section{Author's Contributions}

S. Nilsson, K. Moll, M. Wahlgren, and Q. Chen designed the study. S. Nilsson, K. Moll, D. Angeletti, and L. Albrecht carried out the experiments. I. Kursula was involved in the structural analysis of the protein. S. Nilsson, K. Moll, D. Angeletti, I. Kursula, and L. Albrecht analyzed the data. N. Jiang, X. Sun, and K. Berzins contributed reagents/samples used in the study. S. Nilsson, K. Moll, and Q. Chen wrote the paper. All authors have read and approved the final paper.

\section{Acknowledgments}

The authors thank Professors Hans-Peter Beck and John H. Adams for their kind antibody gifts and Gustav Sundqvist (Royal Institute of Technology, Stockholm) for the mass spectrometric analyses. This study was carried out with support from the European Malaria Consortium (BioMalPar), the Swedish Research Council, SIDA/SAREC, Karolinska Institutet, the National Basic Research Program of China
(973 Program, no. 2007CB513100; QC), and the European Commission FP6 Marie Curie Program (IK). The research leading to these results has received funding from the European Community's Seventh Framework Programme (FP7/2007-2013) under grant agreement $\mathrm{N}^{\circ} 242095$.

\section{References}

[1] WHO, World Malaria Report 2008. Geneva, Switzerland.

[2] J. H. Adams, P. L. Blair, O. Kaneko, and D. S. Peterson, "An expanding ebl family of Plasmodium falciparum," Trends in Parasitology, vol. 17, no. 6, pp. 297-299, 2001.

[3] J. H. Adams, B. K. L. Sim, S. A. Dolan, X. Fang, D. C. Kaslow, and L. H. Miller, "A family of erythrocyte binding-proteins of malaria parasites," Proceedings of the National Academy of Sciences of the United States of America, vol. 89, no. 15, pp. 7085-7089, 1992.

[4] K. Moll, A. Chêne, U. Ribacke et al., "A novel DBL-domain of the P. falciparum 332 molecule possibly involved in erythrocyte adhesion," PLoS ONE, vol. 2, article e477, no. 5, 2007.

[5] B. K. L. Sim, T. Toyoshima, J. D. Haynes, and M. Aikawa, "Localization of the 175-kilodalton erythrocyte binding antigen in micronemes of Plasmodium falciparum merozoites," Molecular and Biochemical Parasitology, vol. 51, no. 1, pp. 157159, 1992.

[6] M. Haeggström, F. Kironde, K. Berzins, Q. S. Chen, M. Wahlgren, and V. Fernandez, "Common trafficking pathway for variant antigens destined for the surface of the Plasmodium falciparum-infected erythrocyte," Molecular and Biochemical Parasitology, vol. 133, no. 1, pp. 1-14, 2004.

[7] K. Hinterberg, A. Scherf, J. Gysin et al., "Plasmodium falciparum: the Pf332 antigen is secreted from the parasite by a brefeldin a-dependent pathway and is translocated to the erythrocyte membrane via the maurer's clefts," Experimental Parasitology, vol. 79, no. 3, pp. 279-291, 1994.

[8] D. Mattei, K. Berzins, M. Wahlgren et al., "Cross-reactive antigenic determinants present on different Plasmodium falciparum blood-stage antigens," Parasite Immunology, vol. 11, no. 1, pp. 15-29, 1989.

[9] D. Mattei and A. Scherf, "The Pf332 gene codes for a megadalton protein of Plasmodium falciparum asexual blood stages," Memorias do Instituto Oswaldo Cruz, vol. 87, pp. 163168, 1992.

[10] D. Mattei and A. Scherf, "The Pf332 gene of Plasmodium falciparum codes for a giant protein that is translocated from the parasite to the membrane of infected erythrocytes," Gene, vol. 110, no. 1, pp. 71-79, 1992.

[11] G. Winter, S. Kawai, M. Haeggström et al., "SURFIN is a polymorphic antigen expressed on Plasmodium falciparum merozoites and infected erythrocytes," Journal of Experimental Medicine, vol. 201, no. 11, pp. 1853-1863, 2005.

[12] J. Iqbal, P. Perlmann, B. M. Greenwood, and K. Berzins, "Seroreactivity with the Plasmodium falciparum blood stage antigen Pf332 in adults and children from malaria-endemic regions," Clinical and Experimental Immunology, vol. 94, no. 1, pp. 68-74, 1993.

[13] A. Kulane, A. B. Siddique, J. L. Sarthou et al., "Human immune responses to the highly repetitive Plasmodium falciparum antigen Pf332," American Journal of Tropical Medicine and Hygiene, vol. 61, no. 1, pp. 141-148, 1999.

[14] N. Ahlborg, D. Haddad, A. B. Siddique et al., "Antibody responses to the repetitive Plasmodium falciparum antigen 
Pf332 in humans naturally primed to the parasite," Clinical and Experimental Immunology, vol. 129, no. 2, pp. 318-325, 2002.

[15] Q. J. Chen, A. Heddini, A. Barragan, V. Fernandez, S. F. A. Pearce, and M. Wahlgren, "The semiconserved head structure of Plasmodium falciparum erythrocyte membrane protein 1 mediates binding to multiple independent host receptors," Journal of Experimental Medicine, vol. 192, no. 1, pp. 1-9, 2000.

[16] J. Söding, A. Biegert, and A. N. Lupas, "The HHpred interactive server for protein homology detection and structure prediction," Nucleic Acids Research, vol. 33, no. 2, pp. W244W248, 2005.

[17] PDB (2010) PDB: Protein Data Bank.

[18] N. H. Tolia, E. J. Enemark, B. K. L. Sim, and L. JoshuaTor, "Structural basis for the EBA-175 erythrocyte invasion pathway of the malaria parasite Plasmodium falciparum," Cell, vol. 122, no. 2, pp. 183-193, 2005.

[19] A. Sali, L. Potterton, F. Yuan, H. van Vlijmen, and M. Karplus, "Evaluation of comparative protein modeling by Modeller," Proteins: Structure, Function and Genetics, vol. 23, no. 3, pp. 318-326, 1995.

[20] C. Gille and C. Frömmel, "STRAP: editor for STRuctural alignments of proteins," Bioinformatics, vol. 17, no. 4, pp. 377378, 2001.

[21] W. DeLano, The PyMOL Molecular Graphics System PyMol 1.3 ed, DeLano Scientific, San Carlos, Calif, USA, 2002.

[22] R. A. Laskowski, M. W. Macarthur, D. S. Moss, and J. M. Thornton, "PROCHECK: a program to check the stereochemical quality of protein structures," Journal of Applied Crystallography, vol. 26, pp. 283-291, 1993.

[23] K. Arnold, L. Bordoli, J. Kopp, and T. Schwede, "The SWISSMODEL workspace: a web-based environment for protein structure homology modelling," Bioinformatics, vol. 22, no. 2, pp. 195-201, 2006.

[24] N. Rasti, F. Namusoke, A. Chêne et al., "Nonimmune immunoglobulin binding and multiple adhesion characterize Plasmodium falciparum-infected erythrocytes of placental origin," Proceedings of the National Academy of Sciences of the United States of America, vol. 103, no. 37, pp. 13795-13800, 2006.

[25] A. Bolad, S. E. Farouk, E. Israelsson et al., "Distinct interethnic differences in immunoglobulin $G$ class/subclass and immunoglobulin $\mathrm{M}$ antibody responses to malaria antigens but not in immunoglobulin $\mathrm{G}$ responses to nonmalarial antigens in sympatric tribes living in West Africa," Scandinavian Journal of Immunology, vol. 61, no. 4, pp. 380-386, 2005.

[26] A. Dolo, D. Modiano, B. Maiga et al., "Difference in susceptibility to malaria between two sympatric ethnic groups in Mali," American Journal of Tropical Medicine and Hygiene, vol. 72, no. 3, pp. 243-248, 2005.

[27] D. Modiano, A. Chiucchiuini, V. Petrarca et al., "Humoral response to Plasmodium falciparum Pf155/ring-infected erythrocyte surface antigen and Pf332 in three sympatric ethnic groups of Burkina Faso," American Journal of Tropical Medicine and Hygiene, vol. 58, no. 2, pp. 220-224, 1998.

[28] D. Modiano, V. Petrarca, B. S. Sirima et al., "Different response to Plasmodium falciparum malaria in West African sympatric ethnic groups," Proceedings of the National Academy of Sciences of the United States of America, vol. 93, no. 23, pp. 1320613211, 1996.

[29] P. E. Okello, W. van Bortel, A. M. Byaruhanga et al., "Variation in malaria transmission intensity in seven sites throughout Uganda," American Journal of Tropical Medicine and Hygiene, vol. 75, no. 2, pp. 219-225, 2006.
[30] J. Normark, D. Nilsson, U. Ribacke et al., "PfEMP1-DBL1 $\alpha$ amino acid motifs in severe disease states of Plasmodium falciparum malaria," Proceedings of the National Academy of Sciences of the United States of America, vol. 104, no. 40, pp. 15835-15840, 2007.

[31] P. F. Beales, B. Brabin, E. Dorman et al., "Severe falciparum malaria," Transactions of the Royal Society of Tropical Medicine and Hygiene, vol. 94, pp. S1-S90, 2000.

[32] M. E. Molyneux, T. E. Taylor, J. J. Wirima, and A. Borgstein, "Clinical features and prognostic indicators in paediatric cerebral malaria: a study of 131 comatose Malawian children," Quarterly Journal of Medicine, vol. 71, no. 265, pp. 441-459, 1989.

[33] K. Moll, I. Ljungström, H. Perlmann, A. Scherf, and M. Wahlgren, Eds., Methods in Malaria Research, ATCC, Manassas, Va, USA, 5th edition, 2008.

[34] N. J. Greenfield, "Using circular dichroism spectra to estimate protein secondary structure," Nature Protocols, vol. 1, no. 6, pp. 2876-2890, 2006.

[35] S. K. Singh, R. Hora, H. Belrhali, C. E. Chitnis, and A. Sharma, "Structural basis for Duffy recognition by the malaria parasite Duffy-binding-like domain," Nature, vol. 439, no. 7077, pp. 741-744, 2006.

[36] D. Mattei, K. Berzins, M. Wahlgren et al., "Cross-reactive antigenic determinants present on different Plasmodium falciparum blood-stage antigens," Parasite Immunology, vol. 11, no. 1, pp. 15-29, 1989.

[37] C. Spycher, N. Klonis, T. Spielmann et al., "MAHRP-1, a novel Plasmodium falciparum histidine-rich protein, binds ferriprotoporphyrin IX and localizes to the Maurer's clefts," Journal of Biological Chemistry, vol. 278, no. 37, pp. 3537335383, 2003.

[38] F. K. Glenister, K. M. Fernandez, L. M. Kats et al., "Functional alteration of red blood cells by a megadalton protein of Plasmodium falciparum," Blood, vol. 113, no. 4, pp. 919-928, 2009.

[39] A. N. Hodder, A. G. Maier, M. Rug et al., "Analysis of structure and function of the giant protein Pf332 in Plasmodium falciparum," Molecular Microbiology, vol. 71, no. 1, pp. 48-65, 2009.

[40] N. Ahlborg, B. W. Flyg, J. Iqbal, P. Perlmann, and K. Berzins, "Epitope specificity and capacity to inhibit parasite growth in vitro of human antibodies to repeat sequences of the Plasmodium falciparum antigen Ag332," Parasite Immunology, vol. 15, no. 7, pp. 391-400, 1993.

[41] N. Ahlborg, J. Iqbal, L. Björk, S. Ståhl, P. Perlmann, and K. Berzins, "Plasmodium falciparum: differential parasite growth inhibition mediated by antibodies to the antigens pf332 and Pf155/RESA," Experimental Parasitology, vol. 82, no. 2, pp. 155-163, 1996.

[42] N. Ahlborg, J. Iqbal, M. Hansson et al., "Immunogens containing sequences from antigen Pf332 induce Plasmodium falciparum-reactive antibodies which inhibit parasite growth but not cytoadherence," Parasite Immunology, vol. 17, no. 7, pp. 341-352, 1995.

[43] R. Udomsangpetch, J. Carlsson, B. Wahlin et al., "Reactivity of the human monoclonal antibody 33G2 with repeated sequences of three distinct Plasmodium falciparum antigens," Journal of Immunology, vol. 142, no. 10, pp. 3620-3626, 1989.

[44] K. L. Waller, L. M. Stubberfield, V. Dubljevic et al., "Interaction of the exported malaria protein Pf332 with the red blood cell membrane skeleton," Biochimica et Biophysica Acta, vol. 1798, no. 5, pp. 861-871, 2010. 
[45] P. Michon, J. R. Stevens, O. Kaneko, and J. H. Adams, "Evolutionary relationships of conserved cysteine-rich motifs in adhesive molecules of malaria parasites," Molecular Biology and Evolution, vol. 19, no. 7, pp. 1128-1142, 2002.

[46] C. Aurrecoechea, J. Brestelli, B. P. Brunk et al., "PlasmoDB:a functional genomic database for malaria parasites," Nucleic Acids Research, vol. 37, no. 1, pp. D539-D543, 2009.

[47] H. A. Balogun, N. M. Vasconcelos, R. Lindberg et al., "Immunogenicity and antigenic properties of Pf332-C231, a fragment of a non-repeat region of the Plasmodium falciparum antigen Pf332," Vaccine, vol. 28, no. 1, pp. 90-97, 2009.

[48] E. Israelsson, H. Balogun, N. M. Vasconcelos et al., "Antibody responses to a C-terminal fragment of the Plasmodium falciparum blood-stage antigen Pf332 in Senegalese individuals naturally primed to the parasite," Clinical and Experimental Immunology, vol. 152, no. 1, pp. 64-71, 2008.

[49] M. J. Gardner, N. Hall, E. Fung et al., "Genome sequence of the human malaria parasite Plasmodium falciparum," Nature, vol. 419, no. 6906, pp. 498-511, 2002.

[50] X. Z. Su, V. M. Heatwole, S. P. Wertheimer et al., "The large diverse gene family var encodes proteins involved in cytoadherence and antigenic variation of Plasmodium falciparuminfected erythrocytes," Cell, vol. 82, no. 1, pp. 89-100, 1995. 


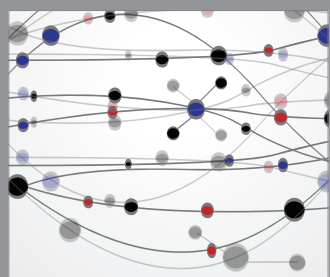

The Scientific World Journal
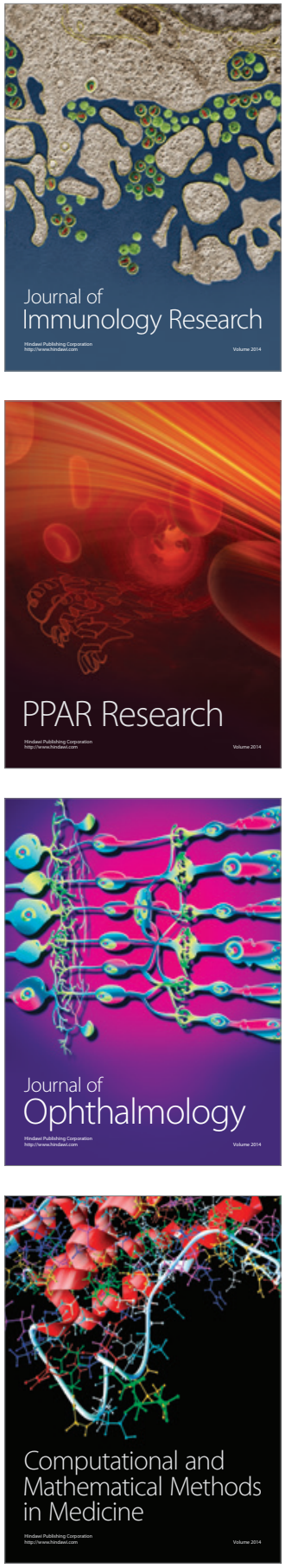

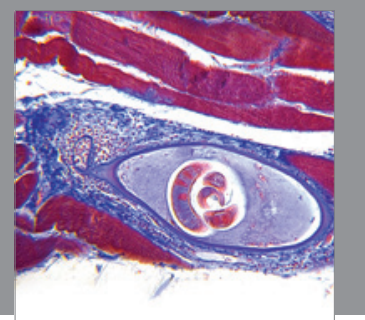

Gastroenterology

Research and Practice
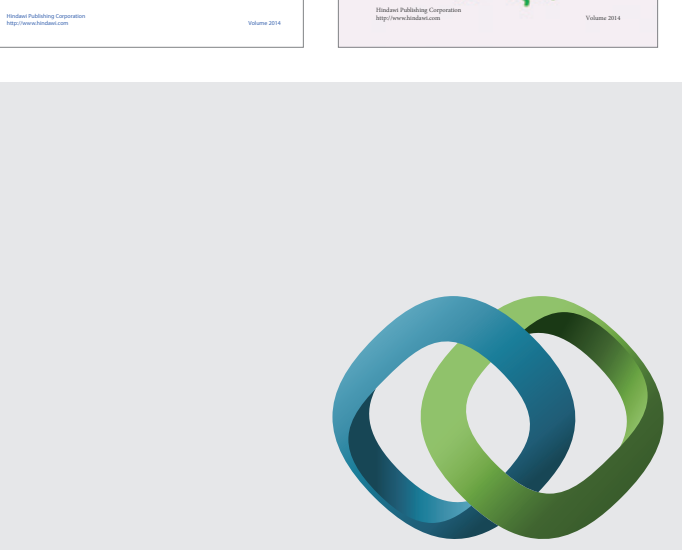

\section{Hindawi}

Submit your manuscripts at

http://www.hindawi.com
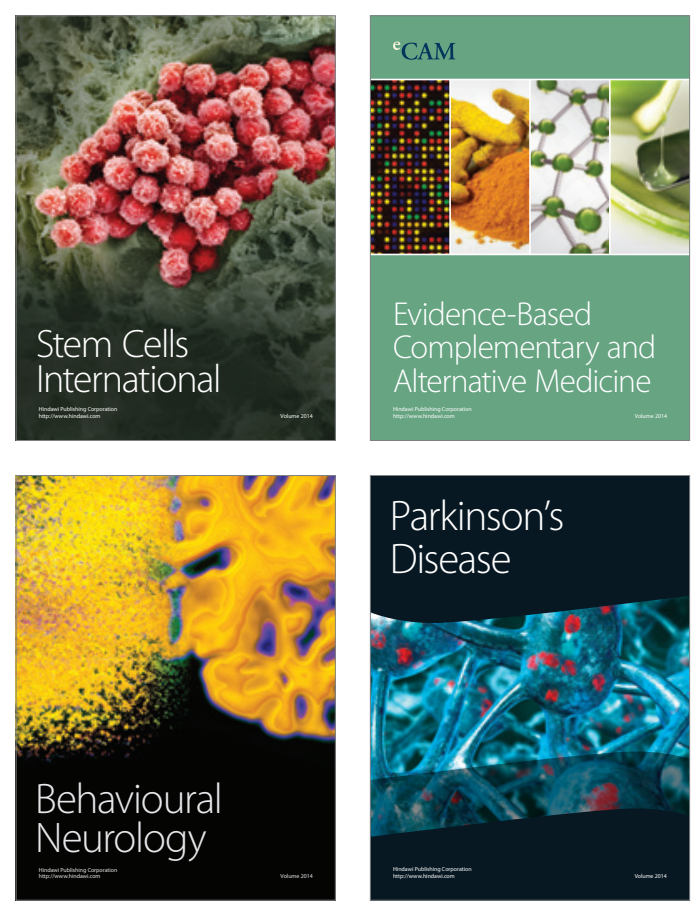

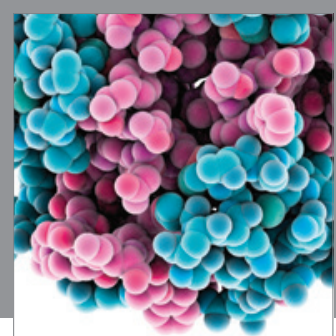

Journal of
Diabetes Research

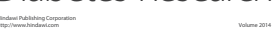

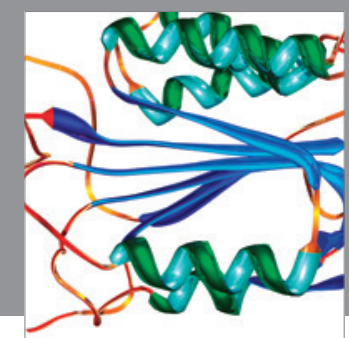

Disease Markers
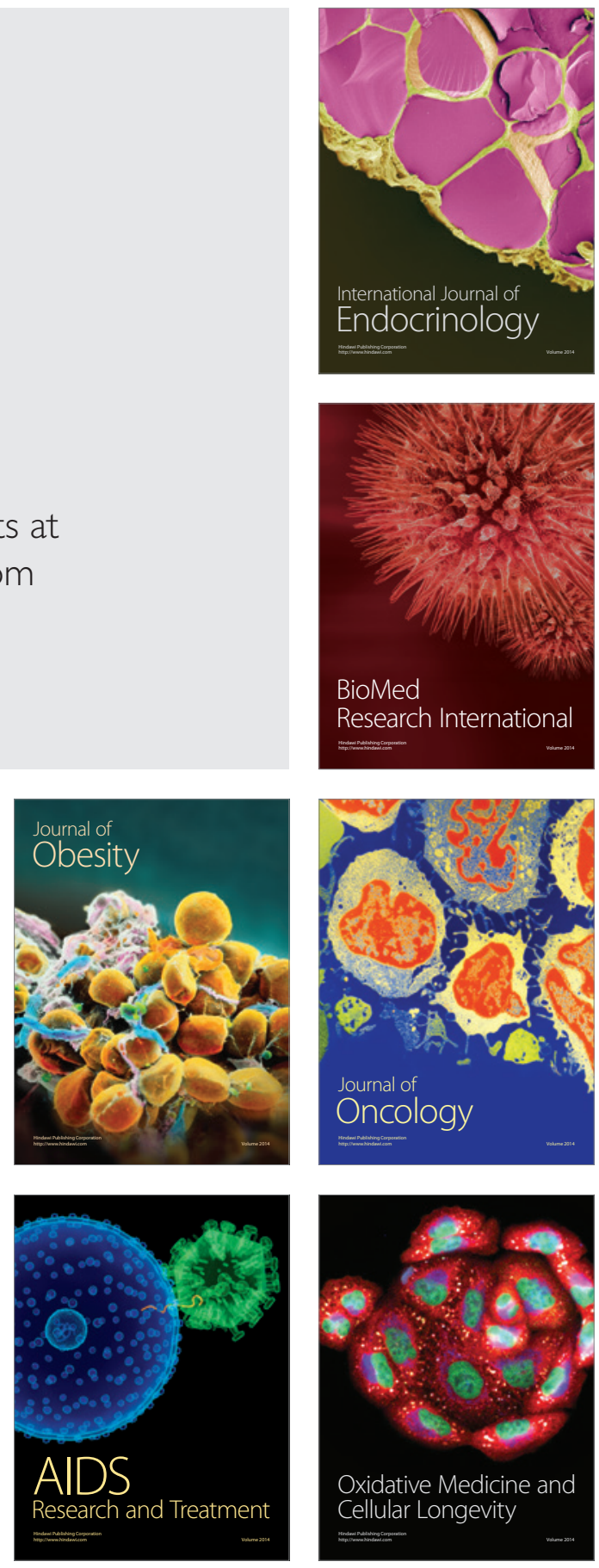